\title{
Haptic Interactions With Multi-Robot Swarms Using Manipulability
}

\author{
Tina Setter, Alex Fouraker, Magnus Egerstedt \\ Georgia Institute of Technology \\ and

\section{Hiroaki Kawashima} \\ Kyoto University
}

This paper investigates how haptic interactions can be defined for enabling a single operator to control and interact with a team of mobile robots. Since there is no unique or canonical mapping from the swarm configuration to the forces experienced by the operator, a suitable mapping must be developed. To this end, multi-agent manipulability is proposed as a potentially useful mapping, whereby the forces experienced by the operator relate to how inputs, injected at precise locations in the team, translate to swarm-level motions. Small forces correspond to directions in which it is easy to move the swarm, while larger forces correspond to more costly directions. Initial experimental results support the viability of the proposed, haptic, human-swarm interaction mapping, through a user study where operators are tasked with driving a collection of robots through a series of way points.

Keywords: Human-robot interaction, multi-agent robotics, haptic force feedback

\section{Introduction}

Multi-agent robotics has matured as a field over the last decade in the sense that a number of different types of control and coordination algorithms have been established for solving a wide range of problems, including area coverage (Bullo, Cortes, \& Martinez, 2009), formation maintenance (Desai, Ostrowski, \& Kumar, 2001; Fax \& Murray, 2002), containment control (Ji, Ferrari-Trecate, Egerstedt, \& Buffa, 2008), tracking of evolving fields (Zhang \& Leonard, 2007), and flocking and swarming (Jadbabaie, Lin, \& Morse, 2003; Olfati-Saber, Fax, \& Murray, 2007), just to name a few. However, what is not clear is how teams of mobile robots can be effectively controlled by human operators.

The current approach to operating autonomous vehicles is one-to-one (i.e., a single operator is interacting with a single vehicle) or even many-to-one (i.e., multiple operators are needed to control a single vehicle, as is often the case when controlling unmanned drones). However, in order to enable a single operator to control and interact with multiple vehicles, new interaction abstractions are needed.

\footnotetext{
Authors retain copyright and grant the Journal of Human-Robot Interaction right of first publication with the work simultaneously licensed under a Creative Commons Attribution License that allows others to share the work with an acknowledgement of the work's authorship and initial publication in this journal.
}

Journal of Human-Robot Interaction, Vol. 4, No. 1, 2015, Pages 60-74. DOI 10.5898/JHRI.4.1.Setter 
Some initial work on human-swarm interactions has been done, and different interaction abstractions have been proposed. For example, McLurkin et al. (2006) investigated how user interfaces should be structured in order to facilitate the control of multi-robot teams. A related type of inquiry concerns the design of useful displays that provide sufficient situational awareness without overloading the operator with data (e.g., Cummings, 2004). Useful abstractions for human-swarm interactions have been studied. In Arkin and Ali (1994), so-called motor schema are defined, and the human operator acts on the team as an additional motor schema. In a similar manner, in Atherton and Goodrich (2009), strengths of biologically inspired entities are being influenced by the user other similar approaches can be found, for example, in Kira and Potter (2009). In Farkhatdinov and Ryu (2008), a switching control strategy is investigated through which a single human operator can sequentially teleoperate multiple robots.

In order to employ haptic interaction modalities for a team of robots, a direct way of mapping the injected control signals onto a force that is experienced by the operator is needed. When interacting with multi-robot teams, the organization of the interaction dynamics matters, as has been observed repeatedly in the literature (e.g., Rahmani, Ji, Mesbahi, \& Egerstedt, 2009; de la Croix \& Egerstedt, 2012). In other words, if the individual robots are nodes in a graph, then interactions between pairs of robots can be encoded through edges between the corresponding nodes in the graph. The resulting graph structure is known as the interaction network (Mesbahi \& Egerstedt, 2010) and its topology (i.e., what the graph looks like) has a direct impact on how easy or hard it is to control the network, as shown in de la Croix and Egerstedt (2012). As a result, the mapping from control inputs to haptic feedback must take the underlying network topology into account.

Manipulability is a standard notion in robotics for describing how effectively joint angle velocities translate into end-effector velocities for high degree-of-freedom manipulators (Bicchi, Melchiorri, \& Balluchi, 1995; Bicchi \& Prattichizzo, 2000; Yoshikawa, 1985). In Kawashima and Egerstedt (2011) and Kawashima and Egerstedt (2014), this idea was moved to the multi-robot domain in the context of "leader-follower" control. In this setup, a subset of the robots are leader robots whose velocities can be controlled directly (corresponding to the manipulator joints), while the remaining agents are the followers (corresponding to the end-effector). In this paper, we pursue this idea as a generator of haptic forces experienced by the operator. That is, high manipulability (swarm is easy to control) yields small forces and low manipulability (swarm is hard to control) yields large forces.

The idea of using haptics to facilitate human interactions with multi-robot teams has been investigated previously. For example, Riedel, Franchi, Giordano, Bülthoff, and Son (2013) used haptic feedback to assist a human operator in controlling multiple UAVs. In this work, a haptic device is used to send the operator information about the presence of obstacles and other external disturbances (such as wind gusts), while also being used by the operator to control the movement of the group of UAVs as a whole. There are other examples where a haptic device has been used to control a group of robots as well as provide feedback to the user. For example, Lee, Martinez-Palafox, and Spong (2005), Lee and Spong (2005), and Rodriguez-Seda et al. (2010) explored bilateral teleoperation of groups of robots by a single master in the presence of communication delays. Similarly, in Secchi, Franchi, Bulthoff, and Robuffo Giordano (2013), a human operator teleoperates a robot team while also controlling the team's degree of connectivity, and haptic feedback is used to inform the operator of the discrepancy between the desired minimum degree of connectivity and the value implemented by the control action. In Nunnally, Walker, Lewis, Chakraborty, and Sycara (2013), the effectiveness of haptic feedback to an operator controlling a robotic swarm was explored through user experiments.

The work we present here differs in that we investigate the effectiveness of different mappings between team-level properties (manipulability) and the haptic feedback force that the operator ex- 
periences when interacting with a select subset of robots in the team, whereas the work done in the aforementioned literature picks one such mapping a priori to relay information to the operator. It also differs in the fact that we are using manipulability to let the human operator know how effectively the leader of the group is controlling the motion of the followers.

The outline of this paper is as follows: In Section 2, we introduce the idea of leader-follower networks of mobile robots and discuss how the notion of a manipulability index can be applied to these networks under a general choice of interaction dynamics. In Section 3, we justify the use of haptics to relay this manipulability information to a human operator that is tasked with controlling a multi-robot team and discuss the different types of mappings between manipulability and haptic force that were explored in this work. In Section 4, the setup of the user experiments are described, and the results comparing these different mappings are presented.

\section{Manipulability and Leader-Follower Interactions}

In this paper, we follow the leader-follower approach to controlling teams of mobile robots, whereby a subset of the robots (i.e., the leaders) are controlled directly, and the control signals are indirectly propagated through the network through the leaders' motions. Since our aim is to provide meaningful and effective haptic feedback to the operator, an instantaneous notion is needed for how easy or hard it is to interact with the robot team. This means that the standard notion of point-to-point controllability may not be ideal, since it is a notion that concerns itself with the (possibly long-term) transfer of the system from one state to another.

In contrast to controllability, which is not an instantaneous property, manipulability is a promising candidate for providing the needed, instantaneous, haptic feedback. As already stated, it is a term borrowed from the robotic manipulation literature (e.g., Bicchi et al., 1995; Bicchi \& Prattichizzo, 2000; Yoshikawa, 1985), and in this section, we recall the main manipulability ideas.

Consider a network consisting of $N$ robots, divided into groups of leaders and followers, such that there are $N_{f}$ followers and $N_{l}$ leaders, with $N_{f}+N_{l}=N$. Also assume that, at time $t$, each robot is located at position $x_{i}(t) \in \mathbb{R}^{d}, i=1, \ldots, N$, where $d$ is the spatial dimension under consideration (e.g., $d=2$ in the case of planar robots, $d=3$ if they move in a three dimensional space, and so forth). We can aggregate the positions together to describe the overall position of the robot team at time $t$, which is given by $x(t)=\left[x_{1}^{T}(t), \ldots, x_{N}^{T}(t)\right]^{T} \in \mathbb{R}^{N d}$. For the sake of notational simplicity, we assume that the indexing of the agents is such that the first $N_{f}$ agents are the followers, and the last $N_{l}$ agents are the leaders. Under this indexing, we have that $x(t)=\left[x_{f}^{T}(t), x_{l}^{T}(t)\right]^{T}$, where $x_{f}(t)=\left[x_{1}^{T}(t), \ldots, x_{N_{f}}^{T}(t)\right]^{T} \in \mathbb{R}^{N_{f} d}$ and $x_{l}(t)=\left[x_{N_{f}+1}^{T}(t), \ldots, x_{N}^{T}(t)\right]^{T} \in \mathbb{R}^{N_{l} d}$.

In a leader-follower network, the idea is to let the leader velocities be controlled by external inputs (the control signals provided by the operator) and then let the follower velocities be defined through pairwise interactions between adjacent agents, as is standard in the multi-robot literature (e.g., Bullo et al., 2009; Mesbahi \& Egerstedt, 2010). As a number of different such pairwise interaction laws have been proposed, our ambition is to design a haptic interaction framework that is largely agnostic to the actual choice of interaction dynamics. However, some choices will have to be made, and we assume that the team of robots is tasked with maintaining desired, pairwise inter-robot distances. This is a rather general version of the so-called formation control problem, and what it means is simply that whenever robots $i$ and $j$ are adjacent in the underlying information-exchange network, their task is to make the distance between them, $\left\|x_{i}-x_{j}\right\|$, as close as possible to a desired distance $d_{i j}$. Not all robots necessarily will be adjacent to all other robots, and thus the formation need not be rigid.

Using this formation-based leader-follower setup, one can formulate the multi-agent manipulability index in a manner that is immediately analogous to the way it is formulated for manipulators, 
namely, as a ratio between the leaders' and the followers' velocities, that is,

$$
M=\frac{\left\|\dot{x}_{f}\right\|^{2}}{\left\|\dot{x}_{l}\right\|^{2}} .
$$

An example of this idea is illustrated in Fig. 1, where the multi-robot network on the left has a lower manipulability than the network on the right due to the fact that the leader-follower velocity ratio is smaller in network (a).

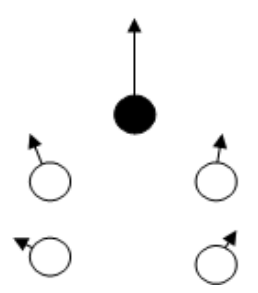

(a) Less Effective

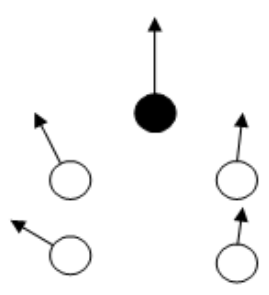

(b) More Effective

Figure 1. Effectiveness of interactions with a leader-follower multi-robot network based on manipulability $\left(N_{l}=1\right)$. The filled circle is the leader and the arrows represent the agents' velocities.

Explicitly untangling what the manipulability index $M$ in Eq. 1 actually depends on is instructive. Clearly, $M$ is a function of $\dot{x}_{l}$, since this is the control signal that is injected by the user. It moreover depends on the total configuration of the multi-robot network (i.e., where all the different robots actually are), $x$, and who they are adjacent to in the network (i.e., what robots are trying to maintain the desired distance from each other). If we let $V=\{1, \ldots, N\}$ denote the set of robots, we can define the set $E \subseteq V \times V$ as the unordered set of robot pairs between which distances are maintained. That the set is unordered means that $(i, j) \in E \Leftrightarrow(j, i) \in E$ (i.e., if robot $i$ cares about its distance to robot $j$, then robot $j$ cares about its distance to robot $i$ ). Using the vertex set $V$ together with the edge set $E$, we have actually specified the undirected graph $G=(V, E)$, that defines the information-exchange network in the multi-robot team. Additionally, the manipulability index in Eq. 1 depends on this graph.

To summarize, we have that $M$ in Eq. 1 depends at the very least on $\dot{x}_{l}, x$, and $G$. Unfortunately, we need one more piece of information in order to compute $M$, namely, $\dot{x}_{f}$. This quantity depends explicitly on the choice of interaction dynamics. However, as our ambition is to be general and not over-design the haptic feedback to a particular choice of interaction dynamics, this obstruction must be remedied. In the manipulation literature, this problem does not arise, since the links in the manipulator are rigid (i.e., there is no interaction dynamics present in the way that it is present in a multi-robot network). Kawashima and Egerstedt (2014) explored how an assumption of rigidity on the links in the multi-robot network translated to a more general and easily computable, yet approximate, manipulability notion that does not depend on the interaction-dynamics, that is,

$$
\tilde{M}\left(x, \dot{x}_{l}, G\right) \approx \frac{\left\|\dot{x}_{f}\right\|^{2}}{\left\|\dot{x}_{l}\right\|^{2}} .
$$

For the sake of composition clarity and for explicitly connecting to the haptic interaction modalities, we here recall the construction from Kawashima and Egerstedt (2014).

To get at a rigid-link approximation of what happens in the multi-robot network without having to explicitly specify the interaction dynamics, we assume that this dynamics is at least able to do 
what it was designed to do (i.e., get close to the desired inter-robot distances sufficiently fast). In other words, for the purpose of obtaining an approximate manipulability measure, we will assume that the desired distances $\left\{d_{i j}\right\}_{\left(v_{i}, v_{j}\right) \in E}$ are perfectly maintained by the followers at all times (i.e., $\left.\left\|x_{i}(t)-x_{j}(t)\right\|=d_{i j}, \forall\left(v_{i}, v_{j}\right) \in E, t \geq 0\right)$. Note that unless the leaders move significantly faster than the followers, this approximation gives a reasonably good characterization of the team behavior under the influence of leader velocity inputs.

Under the rigid-link approximation, the distances between connected robots are static (i.e. they do not change over time). If the trajectories of $x_{i}(t)$ are smooth and differentiable, then this means that

$$
\frac{d}{d t}\left\|x_{i}(t)-x_{j}(t)\right\|^{2}=0, \forall\left(v_{i}, v_{j}\right) \in E, t \geq 0
$$

which expands to

$$
\left(x_{i}-x_{j}\right)^{T}\left(\dot{x}_{i}-\dot{x}_{j}\right)=0, \forall\left(v_{i}, v_{j}\right) \in E,
$$

where we have suppressed the dependence on $t$ for the sake of notational simplicity.

Using Eq. 3, the rigid-link approximation condition can be written in matrix form as

$$
R(x) \dot{x}=0,
$$

where $R(x) \in \mathbb{R}^{|E| \times N d}$ is the so-called rigidity matrix of the system, and where $|E|$ is the cardinality of the edge set. Or, if we explicitly call out the parts contributed by the leaders and the followers,

$$
R(x, G)\left[\begin{array}{c}
\dot{x}_{f} \\
\dot{x}_{l}
\end{array}\right]=\left[R_{f}(x, G) \mid R_{l}(x, G)\right]\left[\begin{array}{c}
\dot{x}_{f} \\
\dot{x}_{l}
\end{array}\right]=0,
$$

where $R_{f} \in \mathbb{R}^{|E| \times N_{f} d}$ and $R_{l} \in \mathbb{R}^{|E| \times N_{l} d}$.

Kawashima and Egerstedt (2014) had shown that this, in turn, implies that the follower velocities can be directly expressed as a function of the leader velocities (as well as $x$ and $G$ ), as

$$
\dot{x}_{f}=-R_{f}^{\dagger}(x, G) R_{l}(x, G) \dot{x}_{l},
$$

where $R_{f}^{\dagger}$ is the Moore-Penrose pseudoinverse of $R_{f}$. This relation gives the prescribed, approximate manipulability measure

$$
\tilde{M}\left(x, \dot{x}_{l}, G\right)=\frac{\dot{x}_{l}^{T} J^{T}(x, G) J(x, G) \dot{x}_{l}}{\left\|\dot{x}_{l}\right\|^{2}},
$$

where $J(x, G)=-R_{f}^{\dagger}(x, G) R_{l}(x, G)$. Returning to the discussion of what these measures should depend on, as well as a desire to not have to depend on the particular choices of interaction law, this approximate manipulability measure is what we will use as a generator of haptic feedback signals.

\section{Haptic Manipulability}

One consequence of the approximate manipulability index is that it is "easier" to move the team of robots in certain directions, and with certain choices of leaders. This observation needs to be formalized in order to map the manipulability index onto a meaningful haptic feedback signal. For example, as shown in Kawashima and Egerstedt (2014), in the single-leader case, the approximate manipulability takes a large value when the direction of the leader's motion coincides with that of the motion of the followers' centroid. In the remainder of this paper, we will focus on the single-leader case, because the experiments consist of a single operator controlling a single leader, which is easy and intuitive for the operator to envision. It should be noted that we are not concerned with finding 
the maximum and minimum values of the approximate manipulability index, per se, but rather with finding a mapping from manipulability to haptic feedback that can help a human operator complete a multi-robot task effectively.

Haptics are a natural choice for conveying manipulability because this method requires no intermediary senses. The mechanism of control actuation is intimately tied to the feedback sensation generated by the haptic device. Since the haptic device can also be used by the human operator to control the leader's velocity, this choice removes complexities that may arise from having two separate mechanisms for controlling the leader and conveying manipulability information from the robot team to the operator. The main idea is that the operator should not have to think about how to apply the manipulability information, because the forces acting on the haptic device should force the operator away from directions that result in low values of manipulability.

In order to apply forces to the haptic device, a mapping between the manipulability of the network and the haptic force must be chosen. By choosing a force mapping that is a monotonically decreasing function of manipulability, the user is encouraged by lesser force to move the network in more manipulable directions while being discouraged by greater force from moving in less manipulable directions. The goal is to encourage the user to move the system in directions of higher manipulability, so the control input is more effective in terms of the response of the multi-robot team.

These mappings should, moreover, be constructed in such a way that the maximum force is returned to the operator when the multi-robot team produces zero manipulability in that direction. Similarly, the operator should feel the minimum amount of force when the input direction produces the greatest manipulability that the system can achieve. Additionally, it can be shown that the maximum approximate manipulability in a leader-follower network with a single leader is equal to the number of followers in the network (i.e., $N_{f}$ ).

In the single-leader case with which we are concerned, $N_{l}=1, R_{l}$ can be expressed in terms of $R_{f}$ as

$$
R_{l}=-R_{f} \tilde{I}_{f}
$$

where $\tilde{I}_{f}=\mathbf{1}_{N_{f}} \otimes I_{d}$, where $\mathbf{1}_{N_{f}}$ is an $N_{f}$-dimensional column vector with $1 \mathrm{~s}$ in all of its entries, $\otimes$ denotes the Kronecker product, and $I_{d}$ denotes the $d \times d$ identity matrix. By substituting this $R_{l}$ into Eq. 6, we get

$$
\dot{x}_{f}=-R_{f}^{\dagger} R_{l} \dot{x}_{l}=R_{f}^{\dagger} R_{f}\left(\mathbf{1}_{N_{f}} \otimes I_{d}\right) \dot{x}_{l}=R_{f}^{\dagger} R_{f}\left(\mathbf{1}_{N_{f}} \otimes \dot{x}_{l}\right) .
$$

Since $R_{f}^{\dagger} R_{f}$ is a projection matrix, we get $\left\|\dot{x}_{f}\right\|^{2} \leq N_{f}\left\|\dot{x}_{l}\right\|^{2}$. Thus, the desired result,

$$
\tilde{M}=\frac{\left\|\dot{x}_{f}\right\|^{2}}{\left\|\dot{x}_{l}\right\|^{2}} \leq N_{f},
$$

follows.

Hence, we know a priori what the maximum manipulability value can be. The minimum force exerted by the haptic device should be zero, so that an operator moving the network in the most manipulable direction should not be encouraged by the haptic device to change directions.

In this paper, we explore two possible such mappings. These were chosen since they are, in a certain sense, canonical in that they recover different aspects of what constitutes a potentially useful mapping. One example of such a mapping is a linear function that inversely maps manipulability to haptic force,

$$
F_{\text {linear }}(\tilde{M})=H\left(1-\frac{\tilde{M}}{N_{f}}\right),
$$


where $H$ is the maximum applicable force of the haptic device, $\tilde{M}$ is the approximate manipulability of the team, and (as before) $N_{f}$ is the number of followers in the network.

This linear map does not encourage high manipulability in a particularly forceful way, and it can be contrasted with an inverse exponential map,

$$
F_{\text {exponential }}(\tilde{M})=H \frac{e^{-\alpha \tilde{M}}-e^{-\alpha N_{f}}}{1-e^{-\alpha N_{f}}} .
$$

Here, $\alpha$ is a parameter that can be changed to adjust the rate of change of the force as a function of manipulability. In the next section, these choices are explored in an experimental setting, where users must solve a multi-robot task using both linear and exponential maps.

\section{Experimental Procedures and Results}

User experiments were performed in order to analyze the effectiveness of different mappings between manipulability and haptic force. Ten subjects voluntarily participated in this study, where each subject was tasked with moving a leader-follower network of differential-drive Khepera III robots between different target locations. This choice of task was driven by the fact that in a number of multi-robot applications, team cohesion was provided by the local coordination and control laws, while high-level objectives, such as target locations or directions, were externally applied.

The velocity of the swarm leader was controlled by the subjects using a PHANTOM Omni haptic device, while manipulability information was relayed to the subject via feedback forces on the haptic device. During each run, the subject was required to direct the leader, and hence the robot team, via the haptic device, to one of the target locations and then to the other target location, in either order. The leader robot was to end up on top of each target location, which was marked with an ' $\mathrm{X}$ ' on the floor. The initial configuration of the robots, along with the marked target locations, can be seen in Fig. 2. The leader is the robot with a white styrofoam object on top of it.

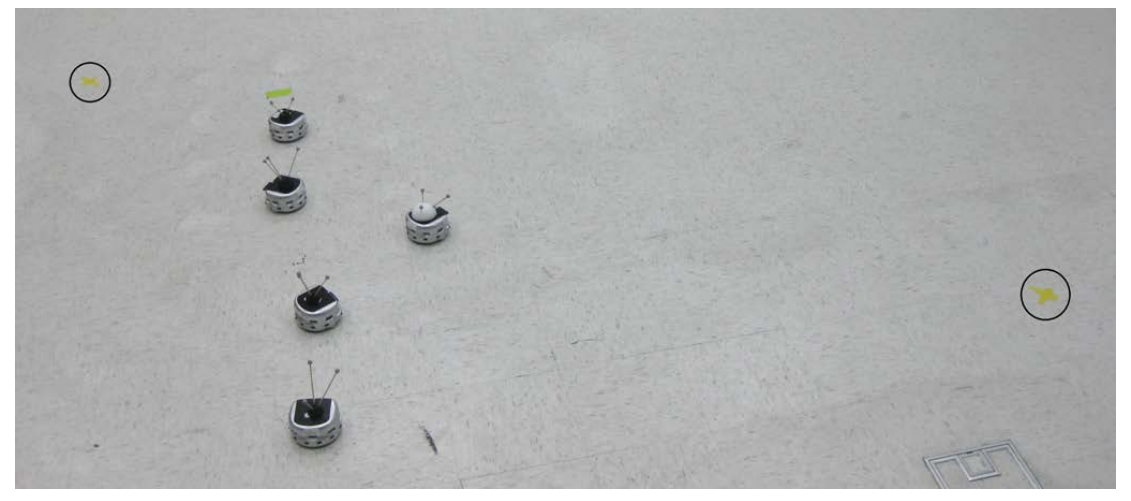

Figure 2. Initial configuration of robots and the two target locations, as illustrated with black circles.

In addition to the physical setup, there was a virtual environment the subject could look at that showed the positions of the robots, the positions of the target locations, and the velocity of the leader (as shown by an arrow with direction and magnitude). This was provided to give the subject a better feel for the environment. A photo of a subject using the haptic device while looking at the virtual environment can be seen in Fig. 3.

The information-exchange network chosen for the user experiments was a line of four followers, with a single leader coming off of the middle two followers, forming a triangle. This configuration 


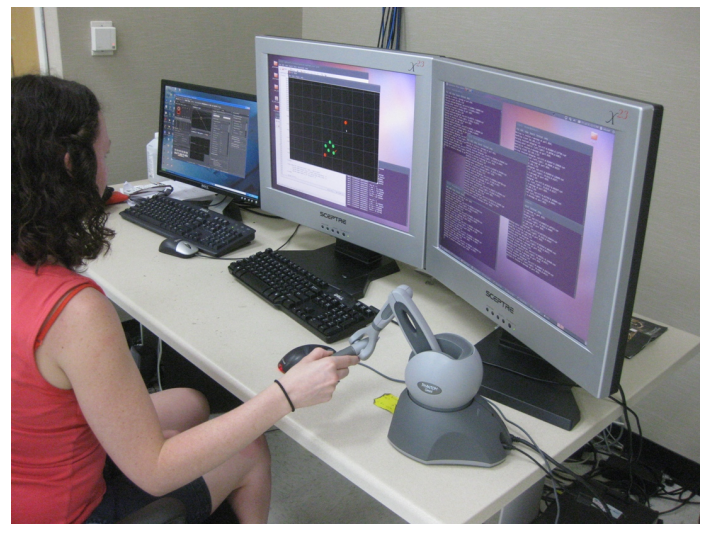

Figure 3. Photo of student utilizing haptic device while looking at the virtual environment (middle screen).

was chosen in order to allow for the network to not be rigid - it could fold and bend while respecting the desired inter-robot distances. This configuration is shown in Fig. 4, where the lines between robots represent links that identify which robots can communicate with each other, or similarly, which robots are in each other's neighborhood set. The leader of the network is represented by a black circle. Each follower robot's control task is to maintain a desired distance between it and the other agents in its neighborhood set. These desired distances are the initial distances between agents, as seen in Fig. 4. Numbering the follower robots in this figure from left to right, it is important to note that robots 1 and 4 each only have one robot in their neighborhood set, being robots 2 and 3 , respectively. This means that the formation shown will not always be maintained, because robots 1 and 4 can move around a bit, as long as robot 1 maintains its distance to robot 2 and robot 3 maintains its distance to robot 4 .

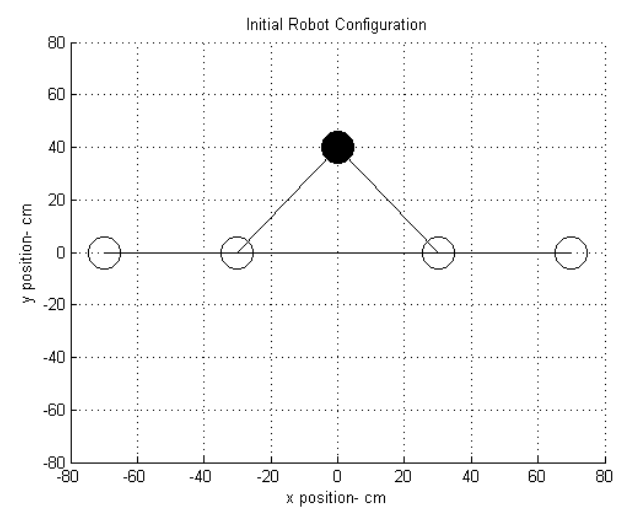

Figure 4. Initial configuration of leader-follower network.

The mappings between manipulability and haptic force used in the user experiments consisted of a linear mapping and four exponential mappings with $\alpha$ parameters of 2, 0.5, -0.5 , and -2 . As discussed previously, these mappings are decreasing functions of manipulability, with a maximum value of one and a minimum value of zero. In order to encourage users to move the leader of the 
network in directions of highest manipulability, the maximum manipulability is mapped to zero haptic force and a manipulability of zero is mapped to a haptic force of one. The maximum value of manipulability is the same as the number of followers in the network, which in this case, is four. The haptic force was applied in the opposite direction of the input velocity that was given by the human operator, so as to be a repulsive force that intends to impede motion in certain directions. These five mappings can be seen in Fig. 5.

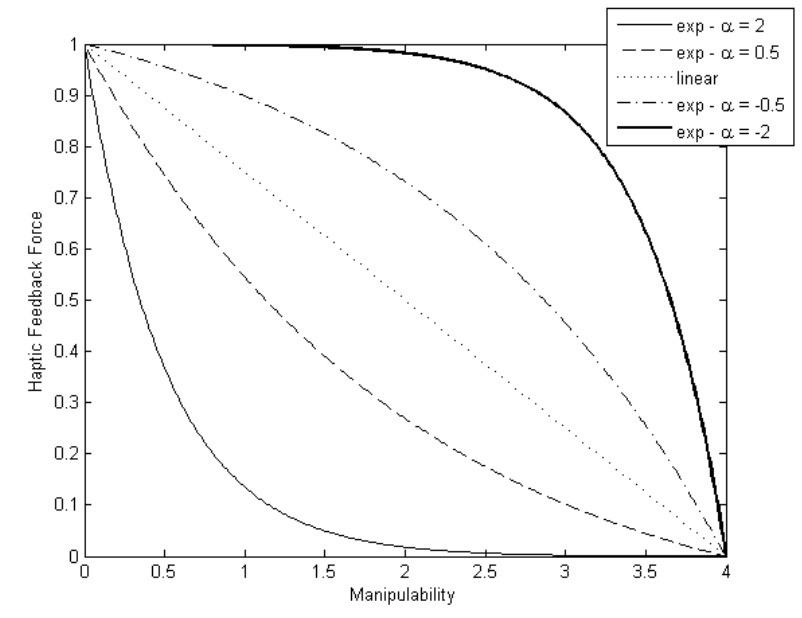

Figure 5. Manipulability vs. haptic force mappings used in user experiments.

It should be pointed out that we did not explicitly test whether or not manipulability is indeed the best notion when interacting with multi-robot teams - both in terms of user experience and in terms of task completion rates. The main objective was to investigate different mappings from manipulability to haptic forces and to gauge their effects on the user experience. As such, the focus is on improving the human experience during the task so the operator can "feel" how easy or hard it is to move the team of robots as a whole. In this experiment, the task is to move the leader between target locations, and it is not a given that manipulability will have a positive effect on task completion. It will, however, let the operator know how effectively the followers' motions are being controlled by the leader's motion, signifying how easy it is to control the entire team.

Each subject performed five runs, of randomized order, with each run using a unique manipulability-haptic force mapping from the five discussed previously. Each run would start with the robots in their initial configuration, as shown in Fig. 2. The subject decided which target location to direct the leader to first and used the haptic device to direct the motion of the leader, and thus the swarm, to this location. When the subject decided that the leader was close enough to the first target location, the subject then directed the leader, via the haptic device, to the second target location. When the subject decided that the leader was close enough to the second target location, the run would end. See Fig. 6 for a photo of the robot team getting close to one of the target locations. The robots were then reset to their initial configuration before the next run would start. After each consecutive run, data was collected and the subject filled out a NASA Task Load Index (TLX) survey, which measured the difficulty of the task.

Using the position and time data collected during the experiments, several measures were computed for comparison. In order to measure how successful the users were in completing the tasks, the shortest distance between the leader and each target location was computed. This distance was 


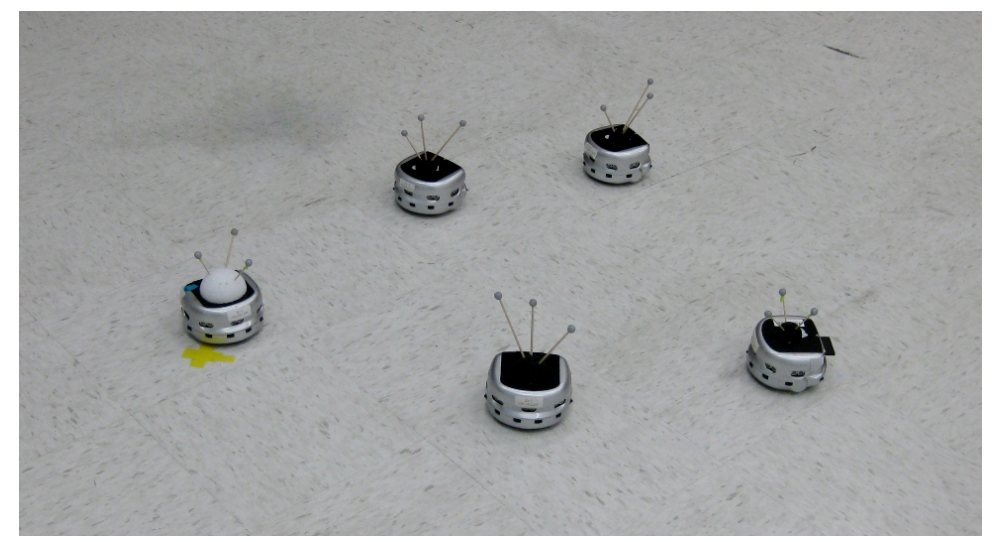

Figure 6. Photo of the robot team approaching one of the target locations during one of the user experiments.

computed for each of the two targets using Eq. 11, where $x_{l}$ is the (2-dimensional) position of the leader and $\tau_{k}$ is the (static) position of the $k$ 'th target location, $k \in 1,2$.

$$
D_{k}=\min _{t \geq 0}\left(\left\|x_{l}(t)-\tau_{k}\right\|\right)
$$

A value of zero for $D_{k}$ means that the leader was precisely on top of the ' $\mathrm{X}$ ' at target location $k$ sometime during a run, whereas greater values indicate that the subject performing the experiment never reached the target location exactly. Smaller values indicate that the subject was able to drive the leader closer to the target location. In addition to the distance from the target locations, the total time that it took to complete both tasks was computed. For purposes of this measure, task completion is defined as the leader being within $15 \mathrm{~cm}$ of the second target location. By averaging these measures across the ten sets of data, it was found that the exponential mapping with $\alpha=0.5$ led to both the shortest task completion time and the shortest distance to target location, for both of the locations. This can be seen in Table 1 .

Table 1: Average task completion time and shortest distance to task location vs. manipulability-haptic force mapping (with standard deviations in parentheses).

\begin{tabular}{|c|c|c|c|c|c|}
\hline & \multicolumn{5}{|c|}{ Manipulability - Force Mapping } \\
\hline & $\alpha=\mathbf{2}$ & $\alpha=\mathbf{0 . 5}$ & Linear & $\alpha=\mathbf{- 0 . 5}$ & $\alpha=\mathbf{- 2}$ \\
\hline \multirow{2}{*}{ Time (sec) } & 85.957 & 84.391 & 115.380 & 87.991 & 87.160 \\
& $(29.553)$ & $(23.489)$ & $(56.554)$ & $(23.880)$ & $(17.796)$ \\
\hline \multirow{2}{*}{$D_{1}(\mathbf{c m})$} & 4.300 & 2.680 & 3.754 & 3.133 & 4.593 \\
& $(2.481)$ & $(1.371)$ & $(3.152)$ & $(1.882)$ & $(1.833)$ \\
\hline \multirow{2}{*}{$D_{2}(\mathbf{c m})$} & 5.047 & 3.574 & 6.745 & 7.581 & 5.977 \\
& $(3.735)$ & $(2.885)$ & $(6.597)$ & $(5.523)$ & $(4.246)$ \\
\hline
\end{tabular}

The last objective measure that was computed and analyzed was the average manipulability of the robot team throughout each of the users' five runs. The ten sets of data were averaged to see how the average manipulability was affected by the haptic mapping and these values can be found in Table 2. It is expected that the mapping with $\alpha=-2$ would be most likely to force the 
user to go in directions with higher manipulability, as the forces are higher under this mapping. Since the mapping with $\alpha=2$ gives off the lowest resistive haptic forces, it was expected that a high manipulability would not be maintained under this mapping. However, the results did not reflect this intuition. The mapping with $\alpha=2$ gave the highest average manipulability of all of the mappings. The other four mappings all had very similar manipulability values.

Table 2: Average manipulability over entire run vs. mapping between manipulability and haptic force (with standard deviations in parentheses).

\begin{tabular}{|c|c|c|c|c|c|}
\hline & \multicolumn{5}{|c|}{ Manipulability - Force Mapping } \\
\hline & $\alpha=\mathbf{2}$ & $\alpha=\mathbf{0 . 5}$ & Linear & $\alpha=\mathbf{- 0 . 5}$ & $\alpha=\mathbf{- 2}$ \\
\hline \multirow{M}{*}{$\tilde{M}$} & 2.6781 & 2.5646 & 2.5695 & 2.5890 & 2.5997 \\
& $(0.3069)$ & $(0.1976)$ & $(0.1754)$ & $(0.2800)$ & $(0.2029)$ \\
\hline
\end{tabular}

This may be due to the nature of the tasks and the initial configuration of the robots. If users were most inclined to move the leader in a direction that happened to have a high manipulability to begin with, then the addition of stronger forces would not make much of a difference. In addition, some users were fixated on the directions that they wanted to move the leader in and were unwilling to let the haptic forces influence their decisions. Perhaps stronger forces would discourage this behavior, but the forces most likely cannot be high enough to impede motion completely due to the limitations of the PHANTOM Omni device. However, these observations are merely speculative and are not supported by the data collected.

The TLX survey required the human subjects to rate each task from 0-100 on six scales: mental demand, physical demand, temporal demand, performance, effort, and frustration. These six measures were then averaged to produce a raw TLX score, where a lower number represents a lower workload required for the task. Each of the ten subjects filled out the survey five times, once for each of the five different manipulability-force mappings. The raw TLX scores can be seen in Fig. 7.

The mean and standard variation of the raw TLX scores for each of the five mappings were computed as given in Table 3 and depicted in Fig. 8. From these results, it can be seen that the exponential mapping with $\alpha=0.5$ produced the best results in terms of workload. It should be noted that the standard deviation values are a bit high and could be due to the fact that the NASA TLX score measures perceived workload, which may vary from person to person.

Table 3: Mean NASA TLX scores for each of the five manipulability-force mappings (with standard deviations in parentheses).

\begin{tabular}{|l|c|c|c|c|c|}
\hline & \multicolumn{5}{|c|}{ Manipulability - Force Mapping } \\
\hline & $\alpha=\mathbf{2}$ & $\alpha=\mathbf{0 . 5}$ & Linear & $\alpha=\mathbf{- 0 . 5}$ & $\alpha=\mathbf{- 2}$ \\
\hline \multirow{2}{*}{ TLX Score } & 16.4167 & 14.0833 & 19.0833 & 19.0000 & 18.6666 \\
& $(14.8087)$ & $(8.8581)$ & $(11.6829)$ & $(11.4786)$ & $(11.4274)$ \\
\hline
\end{tabular}

Based on these results, an exponential mapping with $\alpha=0.5$ outperformed the other four mappings in terms of ease of task and task completion. This particular mapping provides feedback to the user without providing so much force that it makes the task difficult to complete, which may be the case in the $\alpha=-0.5$ and $\alpha=-2$ mappings. Since the goal was to move the leader to a specific set of locations, the user must balance his or her desire to move in a certain direction (toward the 


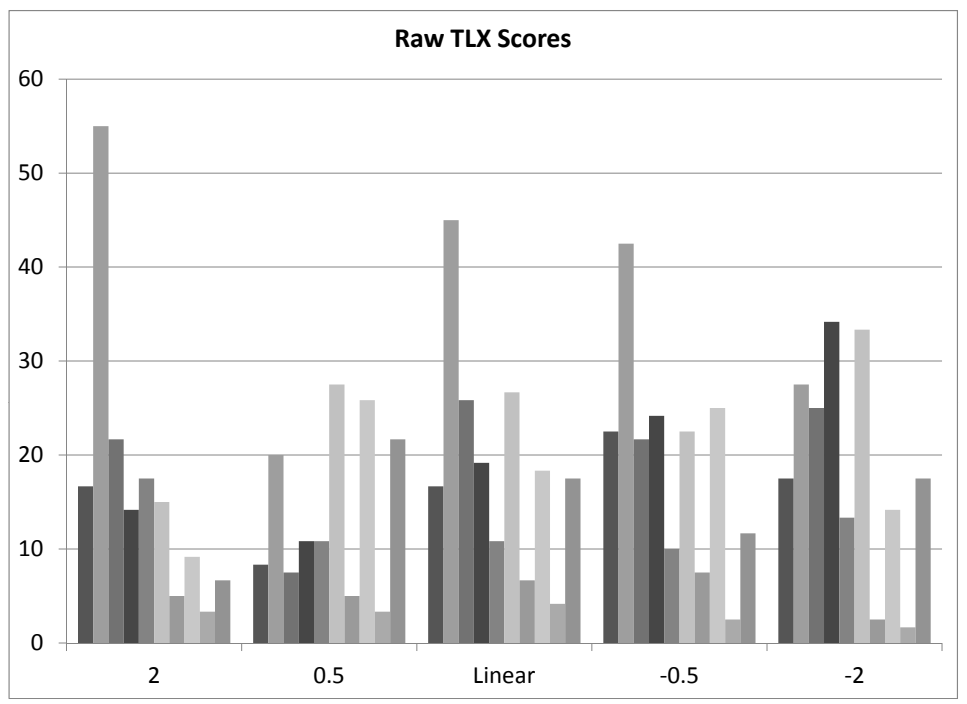

Figure 7. Raw TLX scores given by the subjects for each of the five manipulability-force mappings. For each manipulability-haptic force mapping on the horizontal axis (exponential mapping with $\alpha=2,0.5,-0.5,-2$, and linear mapping), there are ten bars representing the TLX scores given by each of the ten subjects during the user studies, where each subject was tasked with moving a group of robots to two target locations using a haptic device that relays manipulability information about the team of robots using feedback forces. Each subject performed the task five times, where the mapping between manipulability and the haptic feedback force was different for each run. The subjects completed the NASA TLX survey after each run to describe the workload of the task. The TLX scores range from $0-100$, where 0 represents a low workload and 100 represents a high workload.

task location) with the feedback that the haptic controller is giving. Nonetheless, the haptic device provides enough feedback that the user has some intuition about what is going on with the system internally.

\section{Conclusions}

In this paper, we presented the notion of approximate manipulability in leader-follower networks and used this measure to give some intuition about the effectiveness of the control input to a human operator who is controlling the leader of the network through a haptic device. Different mappings between the approximate manipulability of the network and the haptic force applied to the device were explored. If manipulability is chosen as the measure that one wishes to use for providing feedback to a human user, it was found that an exponential mapping with a parameter of $\alpha=0.5$ is preferable to other exponential mappings and to a linear mapping. The experimental results show that the choice of mapping between a particular network property and the force on a haptic device plays a large role when a human operator is given feedback using haptics. In this work, manipulability was chosen as the property of the multi-robot network to be fed back to a human operator, but this can be extended (possibly in future work) to other network properties that a human operator 


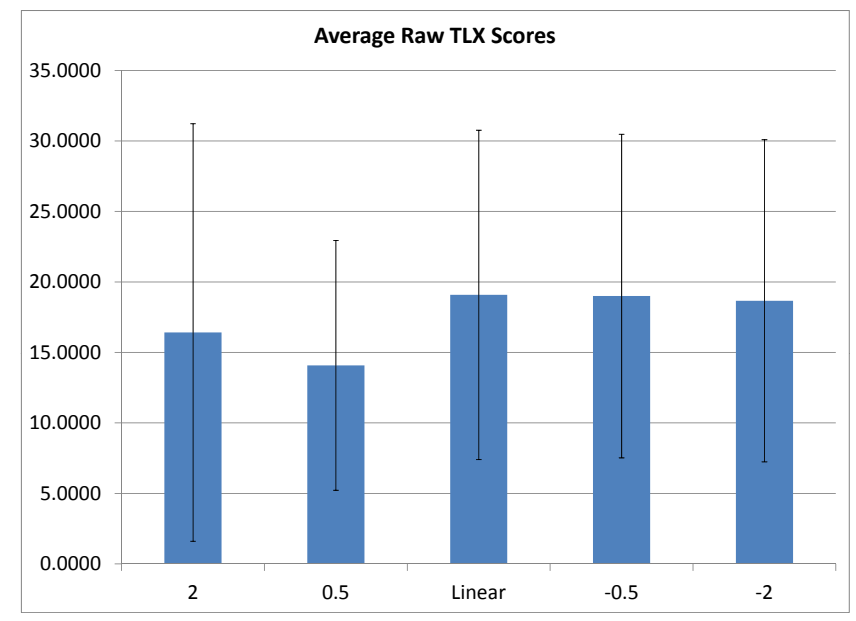

Figure 8. Average of the raw TLX scores given by the subjects for each of the five manipulability-force mappings. The lines above and below the bars show one standard deviation of the data.

may be interested in obtaining via a haptic or other type of interface. This work has shown that there are many ways to map a property of the system being controlled to the haptic force, and in the case of leader-follower network manipulability, the most effective mapping given a particular task was found.

\section{Acknowledgements}

This work was supported in part by a grant from the U.S. Air Force Office of Scientific Research.

\section{References}

Arkin, R., \& Ali, K. (1994). Integration of reactive and telerobotic control in multi- agent robotic systems. In Proceedings of the Third International Conference on Simulation of Adaptive Behavior (p. 473-478). Retrieved from https: //www-robotics.jpl.nasa.gov

Atherton, A., \& Goodrich, M. (2009). Supporting remote and mobile manipulation with an ecological augmented virtuality interface. In Proceedings of the AISB-HRI Symposium on New Frontiers in HumanRobot Interaction.

Bicchi, A., Melchiorri, C., \& Balluchi, D. (1995, Apr). On the mobility and manipulability of general multiple limb robots. IEEE Transactions on Robotics and Automation, 11(2), 215-228. doi:10.1109/70.370503

Bicchi, A., \& Prattichizzo, D. (2000, Aug). Manipulability of cooperating robots with unactuated joints and closed-chain mechanisms. IEEE Transactions on Robotics and Automation, 16(4), 336-345. doi: $10.1109 / 70.864226$

Bullo, F., Cortes, J., \& Martinez, S. (2009). Distributed control of robotic networks. Princeton, NJ: Princeton University Press.

Cummings, M. (2004, June). Human supervisory control of swarming networks. In Proceedings of the Conference on Autonomous Intelligent Networked Systems. Retrieved from http: / / web. mit . edu / 
de la Croix, J., \& Egerstedt, M. (2012). Controllability characterizations of leader-based swarm interactions. In Proceedings of the AAAI Symposium on Human Control of Bio-Inspired Swarms. Retrieved from http://users.ece.gatech.edu/

Desai, J. P., Ostrowski, J. P., \& Kumar, V. (2001). Modeling and control of formations of nonholonomic mobile robots. IEEE Transactions on Robotics and Automation, 17(6), 905-908. doi:10.1109/70.976023

Farkhatdinov, I., \& Ryu, J.-H. (2008, July). Teleoperation of multi-robot and multi-property systems. In Proceedings of the 6th IEEE International Conference on Industrial Informatics (p. 1453-1458). doi: 10.1109/INDIN.2008.4618333

Fax, J. A., \& Murray, R. M. (2002). Graph Laplacians and stabilization of vehicle formations. In Proceedings of the 15th IFAC World Congress (p. 283-288). Retrieved from http: //authors.library.caltech.edu/

Jadbabaie, A., Lin, J., \& Morse, A. S. (2003). Coordination of groups of mobile autonomous agents using nearest neighbor rules. IEEE Transactions on Automatic Control, 48(6), 988-1001. doi: 10.1109/TAC.2003.812781

Ji, M., Ferrari-Trecate, G., Egerstedt, M., \& Buffa, A. (2008, september). Containment control in mobile networks. IEEE Transactions on Automatic Control, 53(8), 1972-1975. doi:10.1109/TAC.2008.930098

Kawashima, H., \& Egerstedt, M. (2011). Approximate manipulability of leader-follower networks. In Proceedings of the IEEE Conference on Decision and Control and European Control Conference (p. 6618-6623). doi:10.1109/CDC.2011.6160974

Kawashima, H., \& Egerstedt, M. (2014). Manipulability of leader-follower networks with the rigid-link approximation. Automatica, 50(3), 695-706. doi:10.1016/j.automatica.2013.11.041

Kira, Z., \& Potter, M. A. (2009). Exerting human control over decentralized robot swarms. In Proceedings of the International Conference on Autonomous Robots and Agents. doi:10.1109/ICARA.2000.4803934

Lee, D., Martinez-Palafox, O., \& Spong, M. (2005, April). Bilateral teleoperation of multiple cooperative robots over delayed communication networks: Application. In Proceedings of the IEEE International Conference on Robotics and Automation (p. 366-371). doi:10.1109/ROBOT.2005.1570146

Lee, D., \& Spong, M. (2005, April). Bilateral teleoperation of multiple cooperative robots over delayed communication networks: Theory. In Proceedings of the IEEE International Conference on Robotics and Automation (p. 360-365). doi:10.1109/ROBOT.2005.1570145

McLurkin, J., Smith, J., Frankel, J., Sotkowitz, D., Blau, D., \& Schmidt, B. (2006, March). Speaking swarmish: Human-robot interface design for large swarms of autonomous mobile robots. In Proceedings of the AAAI Spring Symposium. Stanford, CA, USA. Retrieved from http://mrs l.rice.edu/

Mesbahi, M., \& Egerstedt, M. (2010). Graph theoretic methods in multiagent networks. Princeton, NJ: Princeton University Press.

Nunnally, S., Walker, P., Lewis, M., Chakraborty, N., \& Sycara, K. (2013). Using haptic feedback in human robotic swarms interaction. In Proceedings of the Human Factors and Ergonomics Society (p. 10471051). Pittsburgh, PA: University of Pittsburgh.

Olfati-Saber, R., Fax, J. A., \& Murray, R. M. (2007, Jan). Consensus and cooperation in networked multi-agent systems. Proceedings of the IEEE, 95(1), 215-233. doi:10.1109/JPROC.2006.887293

Rahmani, A., Ji, M., Mesbahi, M., \& Egerstedt, M. (2009). Controllability of multi-agent systems from a graph-theoretic perspective. SIAM Journal on Control and Optimization, 48(1), 162-186. doi: $10.1137 / 060674909$

Riedel, M., Franchi, A., Giordano, P. R., Bülthoff, H. H., \& Son, H. I. (2013). Experiments on intercontinental haptic control of multiple uavs. In Intelligent Autonomous Systems 12 (p. 227-238). Springer. doi: 10.1007/978-3-642-33932-5_22

Rodriguez-Seda, E., Troy, J., Erignac, C., Murray, P., Stipanovic, D., \& Spong, M. (2010, July). Bilateral teleoperation of multiple mobile agents: Coordinated motion and collision avoidance. IEEE Transactions on Control Systems Technology, 18(4), 984-992. doi:10.1109/TCST.2009.2030176

Secchi, C., Franchi, A., Bulthoff, H., \& Robuffo Giordano, P. (2013, May). Bilateral control of the degree of connectivity in multiple mobile-robot teleoperation. In Proceedings of the IEEE International Conference on Robotics and Automation (ICRA), (p. 3645-3652). doi:10.1109/ICRA.2013.6631089 
Yoshikawa, T. (1985). Manipulability of robotic mechanisms. The International Journal of Robotics Research, 4(2), 3-9. doi:10.1177/027836498500400201

Zhang, F., \& Leonard, N. E. (2007). Coordinated patterns of unit speed particles on a closed curve. Systems and Control Letters, 56(6), 397-407. doi:10.1016/j.sysconle.2006.10.027

Tina Setter, School of Electrical and Computer Engineering, Georgia Institute of Technology, Atlanta, GA 30332, USA. Email: tsetter3@gatech.edu. Alex Fouraker, School of Electrical and Computer Engineering, Georgia Institute of Technology, Atlanta, GA 30332, USA. Email: afouraker3@gatech.edu. Hiroaki Kawashima, Graduate School of Informatics, Kyoto University, Kyoto, Japan. Email: kawashima@i.kyoto-u.ac.jp. Magnus Egerstedt, School of Electrical and Computer Engineering, Georgia Institute of Technology, Atlanta, GA 30332, USA. Email: magnus@gatech.edu. 